\title{
Indirect search for dark matter in the Galactic Centre with IceCube
}

\section{The IceCube Collaboration}

(a complete list of authors can be found at the end of the proceedings)

E-mail: nadege.iovine@icecube.wisc.edu

Even though there are strong astrophysical and cosmological indications to support the existence of dark matter, its exact nature remains unknown. We expect dark matter to produce standard model particles when annihilating or decaying, assuming that it is composed of Weakly Interacting Massive Particles (WIMPs). These standard model particles could in turn yield neutrinos that can be detected by the IceCube neutrino telescope. The Milky Way is expected to be permeated by a dark matter halo with an increased density towards its centre. This halo is expected to yield the strongest dark matter annihilation signal at Earth coming from any celestial object, making it an ideal target for indirect searches. In this contribution, we present the sensitivities of an indirect search for dark matter in the Galactic Centre using IceCube data. This low energy dark matter search allows us to cover dark matter masses ranging from $5 \mathrm{GeV}$ to $1 \mathrm{TeV}$. The sensitivities obtained for this analysis show considerable improvements over previous IceCube results in the considered energy range.

Corresponding authors: Nadège Iovine ${ }^{1 *}$, Juan A. Aguilar ${ }^{1}$

${ }^{1}$ Université Libre de Bruxelles

* Presenter

$37^{\text {th }}$ International Cosmic Ray Conference (ICRC 2021)

July 12th-23rd, 2021

Online - Berlin, Germany 


\section{Introduction}

The existence of dark matter is well-supported by a variety of astrophysical and cosmological indications [1,2]. Observations suggest that galaxies are surrounded by a halo of thermal relic dark matter whose density increases towards the centre of the galaxy [3]. Under the assumption that dark matter consists of Weakly Interacting Massive Particles (WIMPs) [4], this high concentration of dark matter at the centre of galaxies would favour the annihilation of dark matter particles. When annihilating or decaying, we expect these dark matter particles to produce standard model particles, which could in turn yield stable particles such as neutrinos. Indirect dark matter experiments, like neutrino telescopes, aim to detect these resulting particles. The presented analysis searches for neutrinos from dark matter self-annihilation in the Galactic Centre using the IceCube [5] detector. With this analysis, the aim is to improve the detection potential for dark matter masses ranging from $5 \mathrm{GeV}$ to $1 \mathrm{TeV}$.

\section{Dark Matter Phenomenology}

We can deduce the expected differential neutrino flux from dark matter self-annihilation in the Galactic Centre from the following equation [6]:

$$
\frac{\mathrm{d} \phi_{v}}{\mathrm{~d} E_{v}}=\frac{1}{4 \pi} \frac{\left\langle\sigma_{\mathrm{A}} v\right\rangle}{2 m_{\mathrm{DM}}^{2}} \frac{\mathrm{d} N_{v}}{\mathrm{~d} E_{v}} J_{\Psi},
$$

where $m_{\mathrm{DM}}$ is the dark matter mass and $\left\langle\sigma_{\mathrm{A}} v\right\rangle$ is the thermally-averaged dark matter self-annihilation cross-section. The differential number of neutrinos produced per annihilating pair of dark matter particles, $\mathrm{d} N_{v} / \mathrm{d} E_{v}$, is taken from the PPPC4 tables [7] and a $100 \%$ branching ratio into either $v_{e} \bar{v}_{e}$, $v_{\mu} \bar{v}_{\mu}, v_{\tau} \bar{v}_{\tau}, W^{+} W^{-}, \mu^{+} \mu^{-} \tau^{+} \tau^{-}$, or $b \bar{b}$ is assumed. These annihilation channels were selected as the corresponding spectra cover a wide range, with the softest spectrum given by $b \bar{b}$ to the hardest spectra given by the $v_{i} \bar{v}_{i}$ channels, where $i$ indicates the neutrino flavour. This analysis also probes dark matter masses ranging from $5 \mathrm{GeV}$ to $1 \mathrm{TeV}$. Neutrino oscillations between the source and the Earth is taken into consideration and the spectra at Earth for all annihilation channels considered and a dark matter mass of $500 \mathrm{GeV}$ can be seen in Figure 1. The J-factor, $J_{\Psi}$, is defined as the integral over the solid angle, $\Delta \Omega$, and line of sight (l.o.s) of the squared dark matter density, $\rho_{\mathrm{DM}}$ :

$$
J_{\Psi}=\int_{\Delta \Omega} \mathrm{d} \Omega(\Psi) \int_{\text {1.o.s }} \rho_{\mathrm{DM}}^{2}(r(l, \Psi)) \mathrm{d} l,
$$

where $\Psi$ is the opening angle to the Galactic Centre. The distribution of dark matter density in the Milky Way can be expressed as a function of the distance, $r$, to the Galactic Centre following [8]:

$$
\rho_{\mathrm{DM}}(r)=\frac{\rho_{0}}{\left(\delta+\frac{r}{r_{\mathrm{s}}}\right)^{\gamma} \cdot\left[1+\left(\frac{r}{r_{\mathrm{s}}}\right)^{\alpha}\right]^{(\beta-\gamma) / \alpha}} .
$$

Values for the normalisation density, $\rho_{0}$, and the scale radius, $r_{s}$, are taken from [9]. As the choice of halo model strongly influences the resulting J-factor, we consider two different dark matter halo model for this analysis. Both models can be expressed by 3 , for which the parameters $(\alpha, \beta, \delta, \gamma)$ are equal to $(2,3,1,1)$ for the Burkert [10] profile and $(1,3,0,1)$ for the Navarro-Frenk-White profile 

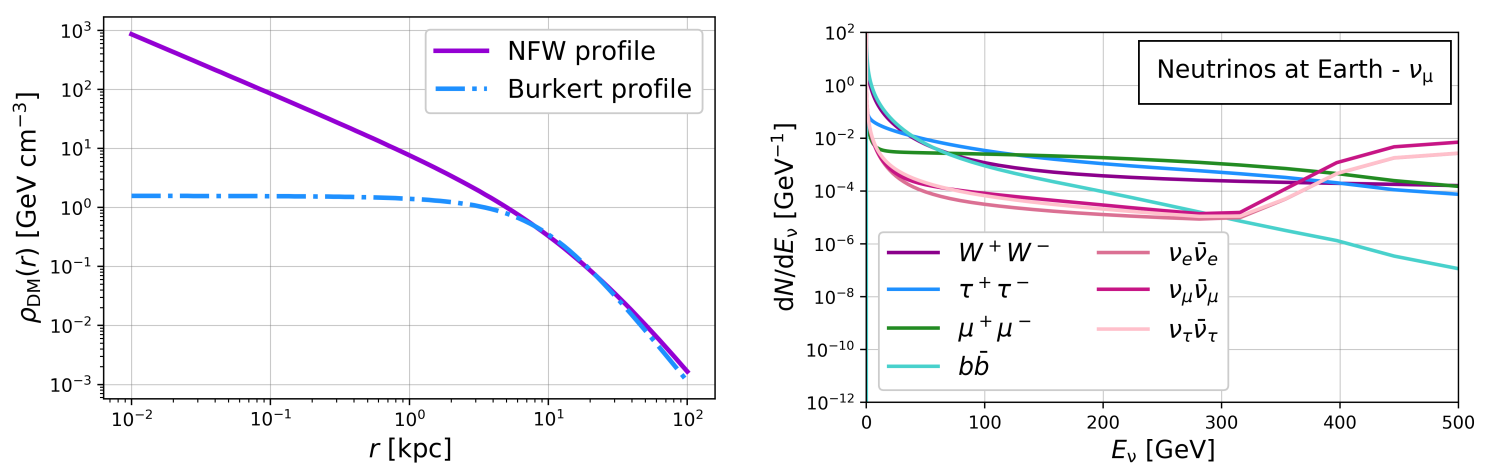

Figure 1: Left: Dark matter density, $\rho_{\mathrm{DM}}$, as a function of the distance to the Galactic Centre, $r$, for the NFW and Burkert profiles. Right: Differential number of muon neutrinos per annihilation at Earth for a dark matter mass of $500 \mathrm{GeV}$.

(NFW) [11]. For "cuspy" dark matter halo profiles, such as the NFW profile, the dark matter density distribution peaks significantly towards the centre of the galaxy, making the signal signature easier to distinguish from the background. As a result, the NFW profile leads to more optimistic sensitivities than core dark matter profiles, such as the Burkert profile. The corresponding dark matter densities can be seen in Figure 1. The J-factors as a function of the opening angle to the Galactic Centre, $\Psi$, are computed using Clumpy [12] for both halo profiles.

\section{Event selection}

Atmospheric muons and neutrinos created by the interactions of cosmic rays in the upper atmosphere represent the main background of IceCube. For up-going events, the Earth acts as a shield against atmospheric muons, which significantly reduces the background. However, when considering sources above the horizon, such as the Galactic Centre, a veto is required in order to reject atmospheric muons. For this analysis, a pre-existing event selection, called oscNext, is considered. This low energy data set is optimised for atmospheric neutrino oscillation measurements. For this event selection, only events recorded within the DeepCore sub-detector [13] are selected, while the remaining parts of the IceCube detector are used as a veto. The oscNext event selection consists of DeepCore events recorded from 2012 to 2020 , for a total livetime of 8.03 years.

\section{Analysis Method}

We consider a binned likelihood method in order to search for an excess of signal neutrinos in the Galactic Centre. This algorithm compares the observed data distribution to expectations based on the background and signal distributions, for each considered combination of dark matter mass, annihilation channel and halo profile. The distributions of interest are used as probability distribution functions (PDFs). For this search, we use three-dimensional PDFs in which the angular information is considered alongside information about the energy and the neutrino flavour of the event. Therefore, the three dimensions of the PDFs are the opening angle to the Galactic Centre $\left(\Psi_{\text {reco }}\right)$, the energy $\left(\log _{10}\left(E_{\text {reco }}\right)\right)$ and the reconstructed neutrino flavour, know as the particle ID 

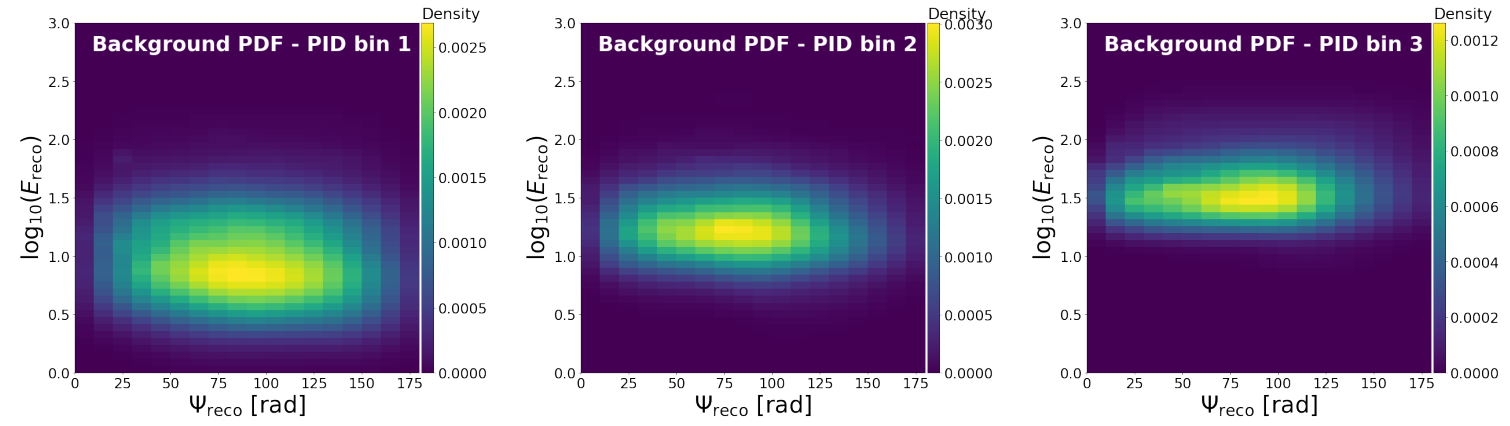

Figure 2: Two-dimensional projection of the background PDF in $\Psi_{\text {reco }}$ and $\log _{10}\left(E_{\text {reco }}\right)$ for each of the 3 PID bins described earlier.

(PID). The background PDF is built from Monte Carlo simulations weighted according to the expected atmospheric flux. The signal PDFs are also constructed from generic neutrino simulations which are weighted with the source morphology and the neutrino spectrum according to Equation 1. A distinct signal PDF is computed for each of the combination of dark matter mass, annihilation channel and halo profile. For the $v_{e} \bar{v}_{e}, v_{\mu} \bar{v}_{\mu}$ and $v_{\tau} \bar{v}_{\tau}$ channels, dark matter masses above $200 \mathrm{GeV}$ are not considered as the signal peaks at $E_{\text {reco }}$ where the contribution of the background is close to zero. In order to avoid such scenario, we introduce a cut restricting the possible parameter combinations. For each combination of dark matter mass, annihilation channel and halo profile, we compute the weighted median of the distribution in reconstructed energy, $E_{\text {reco. }}$. If the resulting median is above the upper bound of the region containing $95 \%$ of the background, the corresponding signal combination is discarded. This is the case for masses above $200 \mathrm{GeV}$ for dark matter annihilation through $v_{i} \bar{v}_{i}$, where $i$ represents the neutrino flavour.

The binning in PID is chosen to optimise the separation between track and cascade events, resulting in 3 PID bins with edges defined as [0, 0.5, 0.85, 1]. Values of the PID close to zero indicates cascade-like events, while values close to one suggest that the event is track-like. This choice of binning thus gives us a first bin containing cascade-like events, a middle bin consisting of a mixture of tracks and cascades, as well as a third bin with track-like events. In order to smooth the background and signal distributions, a Kernel Density Estimation (KDE) is used. To estimate our probability density functions, a Gaussian kernel is thus applied on the $\log \left(\Psi_{\text {reco }}\right)$ and $\log _{10}\left(E_{\text {reco }}\right)$ distributions, for each PID bin. The resulting smoothed distributions are then binned with 18 bins in $\Psi_{\text {reco }}$ ranging from $0^{\circ}$ to $180^{\circ}$ and 50 bins in $\log _{10}\left(E_{\text {reco }}\right)$ between 0 and 3 . The two-dimensional projections of the background PDF for each PID bin can be seen in Figure 2. Similarly, the signal PDF for dark matter particles with a mass of $100 \mathrm{GeV}$ annihilating through the $\tau^{+} \tau^{-}$channel is shown in Figure 3, assuming the NFW halo profile.

The considered likelihood function is built as the product of the Poisson probabilities to observe $n_{\mathrm{obs}}^{i}$ events in a specific bin $i$ :

$$
\mathcal{L}(\mu)=\prod_{i=\text { min }}^{\max } \frac{\left(n_{\mathrm{obs}}^{\mathrm{tot}} f^{i}(\mu)\right)^{n_{\mathrm{obs}}^{i}}}{n_{\mathrm{obs}}^{i} !} e^{-n_{\mathrm{obs}}^{\mathrm{tot}} f^{i}(\mu)},
$$

where $n_{\mathrm{obs}}^{\text {tot }}$ is the total number of events in the sample and $\mu \in[0,1]$ is the fraction of signal events 

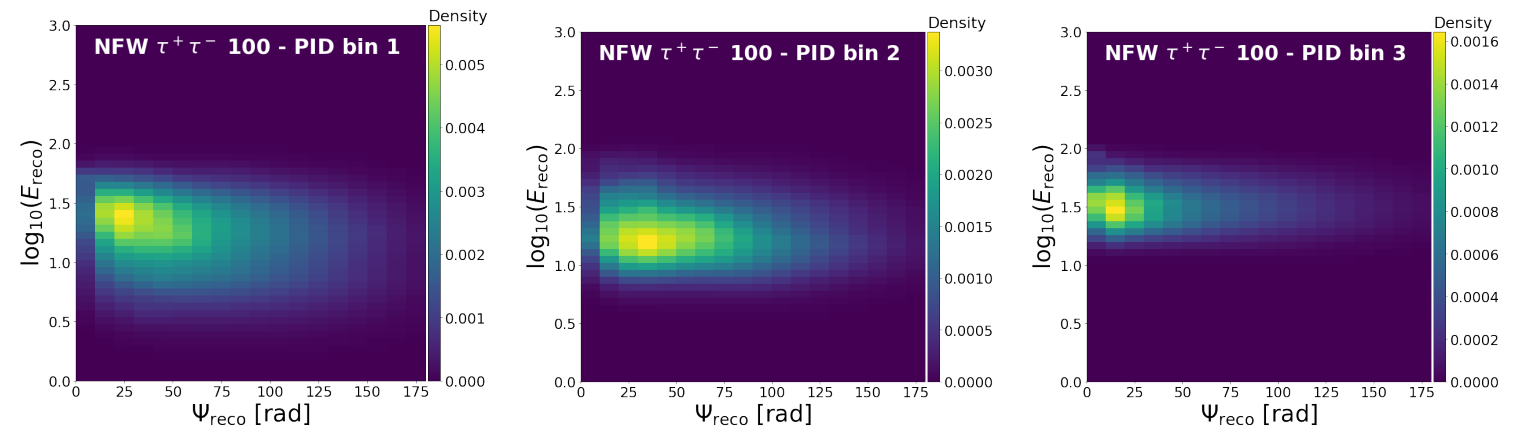

Figure 3: Two-dimensional projection of the signal PDF in $\Psi_{\text {reco }}$ and $\log _{10}\left(E_{\text {reco }}\right)$ for each of the 3 PID bins described earlier, assuming the annihilation of dark matter particles with masses equal to $100 \mathrm{GeV}$ through the $\tau^{+} \tau^{-}$channel for the NFW halo profile.

of the total sample. The fraction of events within a bin $i$ is expressed as:

$$
f^{i}(\mu)=\mu f_{s}^{i}+(1-\mu) f_{\mathrm{bg}}^{i}
$$

where $f_{\text {bg }}$ and $f_{s}$ are the background and signal PDFs. The best estimate of the signal fraction, $\mu_{\text {best }}$, is obtained by maximising the likelihood, $\mathcal{L}(\mu)$. If this value is consistent with the backgroundonly hypothesis, the upper limit on the signal fraction at the $90 \%$ confidence level (CL), $\mu_{90}$, is computed according to the likelihood interval method [14]. The 90\% CL sensitivity is computed by generating 100,000 pseudo-experiments sampled from the background-only PDF. We quote as $90 \%$ CL sensitivity, $\hat{\mu}_{90}$, the median value of the upper limits obtained for each of these pseudoexperiments. From this, we can deduce the sensitivity on the thermally-averaged dark matter self-annihilation cross-section, $\left\langle\sigma_{\mathrm{A}} v\right\rangle$, using the total number of observed events and the estimated number of events for a specific combination of dark matter mass, annihilation channel and halo profile.

\section{Sensitivities}

In this section, we presented the $90 \%$ CL sensitivities on the thermally-averaged dark matter self-annihilation cross-section, $\left\langle\sigma_{\mathrm{A}} v\right\rangle$, obtained for this analysis. These $90 \% \mathrm{CL}$ sensitivities are shown in Figure 4 as a function of the dark matter mass for all evaluated annihilation channels and both considered halo profiles. The sensitivities obtained for this analysis show considerable improvement with respect to previous IceCube results [15]. This enhancement can be seen in Figure 5 for the $\tau^{+} \tau^{-}$annihilation channel and the NFW halo profile. This improvement is due to multiple factors. First, more years of data were considered for this analysis compared to the 3 years of DeepCore previously used. Furthermore, the event selection considered present a considerable improvement when compared to the previously used data set, especially at the lowest energies. Finally, this analysis is including information about the energy and the neutrino flavour along with the angular information, which was not the case for previous IceCube analyses. 

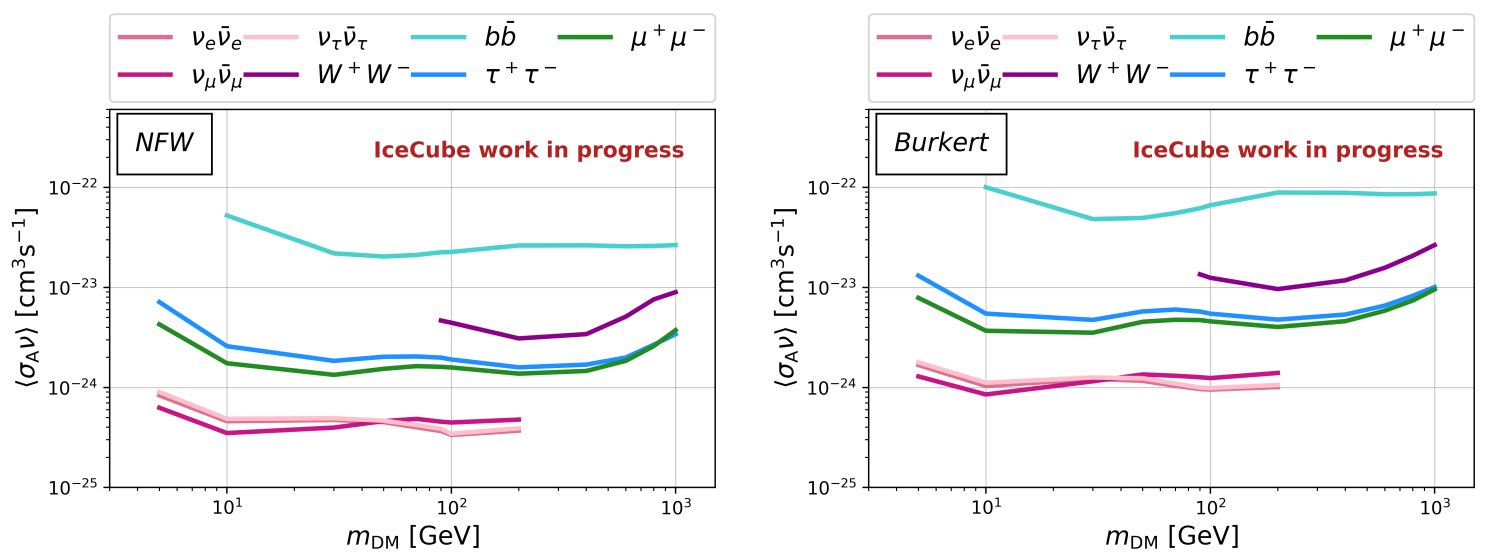

Figure 4: Sensitivities on the thermally-averaged dark matter self-annihilation cross-section $\left\langle\sigma_{\mathrm{A}} v\right\rangle$ as a function of the dark matter mass $m_{\mathrm{DM}}$. All annihilation channels considered for this analysis are presented for both the NFW (left) and Burkert (right) halo profiles.

\section{Conclusion and outlooks}

We computed the sensitivities on $\left\langle\sigma_{\mathrm{A}} v\right\rangle$ for a dark matter search in the Galactic Centre using 8 years of DeepCore data. The obtained sensitivities show considerable improvements when compared to previous IceCube results from similar searches. This improvement is mainly due to the enhanced event selection considered, as well as the inclusion of the energy and the flavour information in the event distributions. As this analysis is in its final state, the final official results should soon be available. If no signal neutrinos were to be found, limits on the thermally-averaged self-annihilation cross-section $\left\langle\sigma_{\mathrm{A}} v\right\rangle$ will be computed.

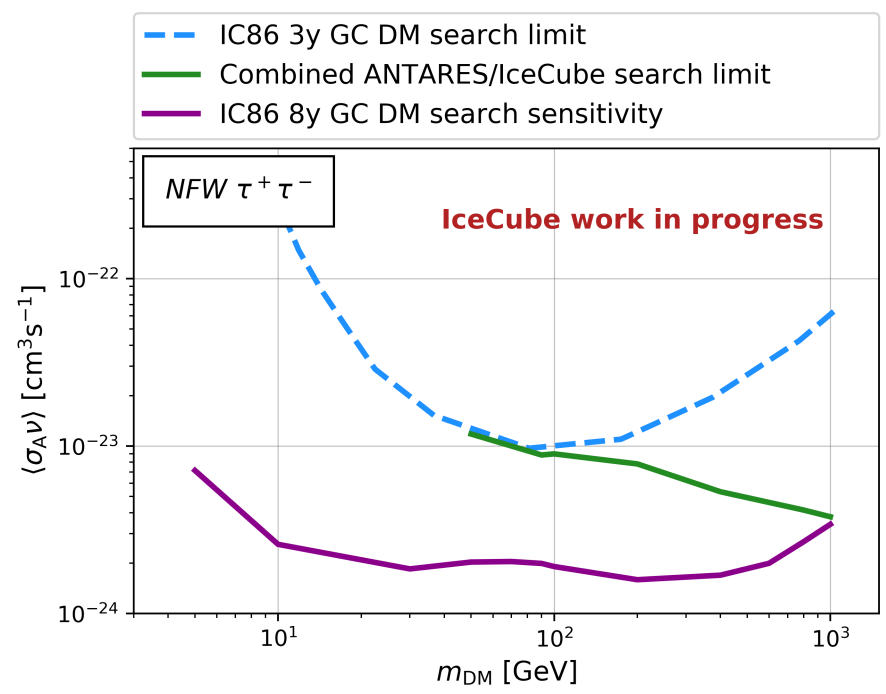

Figure 5: Sensitivity on the thermally-averaged dark matter self-annihilation cross-section $\left\langle\sigma_{\mathrm{A}} v\right\rangle$ for annihilation through $\tau^{+} \tau^{-}$and assuming the NFW halo profile shown along with limits from IceCube [15] and the combined dark matter conducted with ANTARES and IceCube [16] 


\section{References}

[1] G. Bertone and D. Hooper Rev. Mod. Phys. 90 no. 4, (2018) 045002.

[2] Planck Collaboration, P. A. R. Ade et al. Astron. Astrophys. 571 (2014) A16.

[3] A. V. Kravtsov, A. A. Klypin, J. S. Bullock, and J. R. Primack Astrophys. J. 502 (1998) 48.

[4] G. Steigman and M. S. Turner Nucl. Phys. B 253 (1985) 375-386.

[5] IceCube Collaboration, M. G. Aartsen et al. JINST 12 no. 03, (2017) P03012.

[6] H. Yuksel, S. Horiuchi, J. F. Beacom, and S. Ando Phys. Rev. D 76 (2007) 123506.

[7] M. Cirelli, G. Corcella, A. Hektor, G. Hutsi, M. Kadastik, P. Panci, M. Raidal, F. Sala, and A. Strumia JCAP 03 (2011) 051. [Erratum: JCAP 10, E01 (2012)].

[8] J. An and H. Zhao Mon. Not. Roy. Astron. Soc. 428 (2013) 2805-2811.

[9] F. Nesti and P. Salucci JCAP 07 (2013) 016.

[10] A. Burkert Astrophys. J. Lett. 447 (1995) L25.

[11] J. F. Navarro, C. S. Frenk, and S. D. M. White Astrophys. J. 462 (1996) 563-575.

[12] M. Hütten, C. Combet, and D. Maurin Comput. Phys. Commun. 235 (2019) 336-345.

[13] IceCube Collaboration, R. Abbasi et al. Astropart. Phys. 35 (2012) 615-624.

[14] G. Cowan, K. Cranmer, E. Gross, and O. Vitells Eur. Phys. J. C 71 (2011) 1554. [Erratum: Eur.Phys.J.C 73, 2501 (2013)].

[15] IceCube Collaboration, M. G. Aartsen et al. Eur. Phys. J. C 77 no. 9, (2017) 627.

[16] ANTARES, IceCube Collaboration, A. Albert et al. Phys. Rev. D 102 no. 8, (2020) 082002. 


\section{Full Author List: IceCube Collaboration}

R. Abbasi ${ }^{17}$, M. Ackermann ${ }^{59}$, J. Adams ${ }^{18}$, J. A. Aguilar ${ }^{12}$, M. Ahlers ${ }^{22}$, M. Ahrens ${ }^{50}$, C. Alispach ${ }^{28}$, A. A. Alves Jr. ${ }^{31}$, N. M. $\mathrm{Amin}^{42}$, R. An ${ }^{14}$, K. Andeen ${ }^{40}$, T. Anderson ${ }^{56}$, G. Anton ${ }^{26}$, C. Argüelles ${ }^{14}$, Y. Ashida ${ }^{38}$, S. Axani ${ }^{15}$, X. Bai ${ }^{46}$, A. Balagopal V. ${ }^{38}$, A. Barbano ${ }^{28}$, S. W. Barwick ${ }^{30}$, B. Bastian ${ }^{59}$, V. Basu ${ }^{38}$, S. Baur ${ }^{12}$, R. Bay ${ }^{8}$, J. J. Beatty ${ }^{20,21}$, K.-H. Becker ${ }^{58}$, J. Becker Tjus ${ }^{11}$, C. Bellenghi $^{27}$, S. BenZvi ${ }^{48}$, D. Berley ${ }^{19}$, E. Bernardini ${ }^{59}, 60$, D. Z. Besson ${ }^{34,61}$, G. Binder ${ }^{8,9}$, D. Bindig ${ }^{58}$, E. Blaufuss ${ }^{19}$, S. Blot ${ }^{59}$, M. Boddenberg ${ }^{1}$, F. Bontempo ${ }^{31}$, J. Borowka ${ }^{1}$, S. Böser ${ }^{39}$, O. Botner ${ }^{57}$, J. Böttcher ${ }^{1}$, E. Bourbeau ${ }^{22}$, F. Bradascio ${ }^{59}$, J. Braun ${ }^{38}$, S. Bron $^{28}$, J. Brostean-Kaiser ${ }^{59}$, S. Browne ${ }^{32}$, A. Burgman ${ }^{57}$, R. T. Burley ${ }^{2}$, R. S. Busse ${ }^{41}$, M. A. Campana ${ }^{45}$, E. G. Carnie-Bronca ${ }^{2}$, C. $\mathrm{Chen}^{6}$, D. Chirkin ${ }^{38}$, K. Choi ${ }^{52}$, B. A. Clark ${ }^{24}$, K. Clark ${ }^{33}$, L. Classen ${ }^{41}$, A. Coleman ${ }^{42}$, G. H. Collin ${ }^{15}$, J. M. Conrad ${ }^{15}$, P. Coppin $^{13}$, P. Correa ${ }^{13}$, D. F. Cowen ${ }^{55}, 56$, R. Cross $^{48}$, C. Dappen ${ }^{1}$, P. Dave ${ }^{6}$, C. De Clercq ${ }^{13}$, J. J. DeLaunay ${ }^{56}$, H. Dembinski ${ }^{42}$, K. Deoskar $^{50}$, S. De Ridder ${ }^{29}$, A. Desai ${ }^{38}$, P. Desiati ${ }^{38}$, K. D. de Vries ${ }^{13}$, G. de Wasseige ${ }^{13}$, M. de With ${ }^{10}$, T. DeYoung ${ }^{24}$, S. Dharani ${ }^{1}$, A. Diaz ${ }^{15}$, J. C. Díaz-Vélez ${ }^{38}$, M. Dittmer ${ }^{41}$, H. Dujmovic ${ }^{31}$, M. Dunkman ${ }^{56}$, M. A. DuVernois ${ }^{38}$, E. Dvorak ${ }^{46}$, T. Ehrhardt ${ }^{39}$, P. Eller $^{27}$, R. Engel $^{31,32}$, H. Erpenbeck ${ }^{1}$, J. Evans ${ }^{19}$, P. A. Evenson ${ }^{42}$, K. L. Fan ${ }^{19}$, A. R. Fazely ${ }^{7}$, S. Fiedlschuster ${ }^{26}$, A. T. Fienberg ${ }^{56}$, K. Filimonov ${ }^{8}$, C. Finley ${ }^{50}$, L. Fischer ${ }^{59}$, D. Fox ${ }^{55}$, A. Franckowiak ${ }^{11,59}$, E. Friedman ${ }^{19}$, A. Fritz ${ }^{39}$, P. Fürst ${ }^{1}$, T. K. Gaisser ${ }^{42}$, J. Gallagher $^{37}$, E. Ganster ${ }^{1}$, A. Garcia ${ }^{14}$, S. Garrappa ${ }^{59}$, L. Gerhardt ${ }^{9}$, A. Ghadimi ${ }^{54}$, C. Glaser ${ }^{57}$, T. Glauch ${ }^{27}$, T. Glüsenkamp ${ }^{26}$, A. Goldschmidt $^{9}$, J. G. Gonzalez ${ }^{42}$, S. Goswami ${ }^{54}$, D. Grant ${ }^{24}$, T. Grégoire ${ }^{56}$, S. Griswold ${ }^{48}$, M. Gündüz ${ }^{11}$, C. Günther ${ }^{1}$, C. Haack ${ }^{27}$,

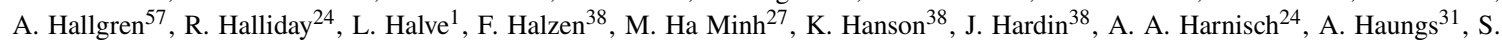
Hauser $^{1}$, D. Hebecker ${ }^{10}$, K. Helbing ${ }^{58}$, F. Henningsen ${ }^{27}$, E. C. Hettinger ${ }^{24}$, S. Hickford ${ }^{58}$, J. Hignight ${ }^{25}$, C. Hill ${ }^{16}$, G. C. Hill ${ }^{2}$, K. D.

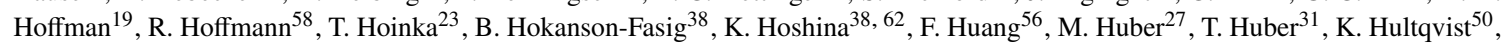
M. Hünnefeld ${ }^{23}$, R. Hussain ${ }^{38}$, S. In ${ }^{52}$, N. Iovine ${ }^{12}$, A. Ishihara ${ }^{16}$, M. Jansson ${ }^{50}$, G. S. Japaridze ${ }^{5}$, M. Jeong ${ }^{52}$, B. J. P. Jones ${ }^{4}$, D. Kang ${ }^{31}$, W. Kang ${ }^{52}$, X. Kang ${ }^{45}$, A. Kappes ${ }^{41}$, D. Kappesser ${ }^{39}$, T. Karg ${ }^{59}$, M. Karl ${ }^{27}$, A. Karle ${ }^{38}$, U. Katz ${ }^{26}$, M. Kauer ${ }^{38}$, M. Kellermann ${ }^{1}$, J. L. Kelley $^{38}$, A. Kheirandish ${ }^{56}$, K. Kin ${ }^{16}$, T. Kintscher ${ }^{59}$, J. Kiryluk ${ }^{51}$, S. R. Klein ${ }^{8,9}$, R. Koirala ${ }^{42}$, H. Kolanoski ${ }^{10}$, T. Kontrimas ${ }^{27}$, L. $\mathrm{Köpke}^{39}$, C. Kopper ${ }^{24}$, S. Kopper ${ }^{54}$, D. J. Koskinen ${ }^{22}$, P. Koundal ${ }^{31}$, M. Kovacevich ${ }^{45}$, M. Kowalski ${ }^{10,59}$, T. Kozynets ${ }^{22}$, E. Kun ${ }^{11}$, N. Kurahashi ${ }^{45}$, N. Lad ${ }^{59}$, C. Lagunas Gualda ${ }^{59}$, J. L. Lanfranchi ${ }^{56}$, M. J. Larson ${ }^{19}$, F. Lauber ${ }^{58}$, J. P. Lazar ${ }^{14,38}$, J. W. Lee ${ }^{52}$, K. Leonard $^{38}$, A. Leszczyńska ${ }^{32}$, Y. Li ${ }^{56}$, M. Lincetto ${ }^{11}$, Q. R. Liu ${ }^{38}$, M. Liubarska ${ }^{25}$, E. Lohfink ${ }^{39}$, C. J. Lozano Mariscal ${ }^{41}$, L. Lu ${ }^{38}$,

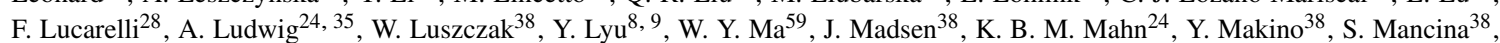

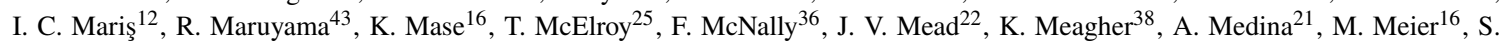
Meighen-Berger $^{27}$, J. Micallef ${ }^{24}$, D. Mockler ${ }^{12}$, T. Montaruli ${ }^{28}$, R. W. Moore ${ }^{25}$, R. Morse ${ }^{38}$, M. Moulai ${ }^{15}$, R. Naab ${ }^{59}$, R. Nagai ${ }^{16}$, U. Naumann $^{58}$, J. Necker ${ }^{59}$, L. V. Nguyễn ${ }^{24}$, H. Niederhausen ${ }^{27}$, M. U. Nisa ${ }^{24}$, S. C. Nowicki ${ }^{24}$, D. R. Nygren ${ }^{9}$, A. Obertacke Pollmann ${ }^{58}$, M. Oehler ${ }^{31}$, A. Olivas ${ }^{19}$, E. O'Sullivan ${ }^{57}$, H. Pandya ${ }^{42}$, D. V. Pankova ${ }^{56}$, N. Park ${ }^{33}$, G. K. Parker ${ }^{4}$, E. N. Paudel ${ }^{42}$, L. Paul ${ }^{40}$, C. Pérez de los $\operatorname{Heros}^{57}$, L. Peters ${ }^{1}$, J. Peterson ${ }^{38}$, S. Philippen ${ }^{1}$, D. Pieloth ${ }^{23}$, S. Pieper ${ }^{58}$, M. Pittermann ${ }^{32}$, A. Pizzuto ${ }^{38}$, M. Plum ${ }^{40}$, Y. Popovych $^{39}$, A. Porcelli ${ }^{29}$, M. Prado Rodriguez ${ }^{38}$, P. B. Price ${ }^{8}$, B. Pries ${ }^{24}$, G. T. Przybylski ${ }^{9}$, C. Raab ${ }^{12}$, A. Raissi ${ }^{18}$, M. Rameez ${ }^{22}$, K. $^{2}$. Rawlins $^{3}$, I. C. Rea ${ }^{27}$, A. Rehman ${ }^{42}$, P. Reichherzer ${ }^{11}$, R. Reimann ${ }^{1}$, G. Renzi ${ }^{12}$, E. Resconi ${ }^{27}$, S. Reusch ${ }^{59}$, W. Rhode ${ }^{23}$, M. Richman $^{45}$, B. Riedel ${ }^{38}$, E. J. Roberts ${ }^{2}$, S. Robertson ${ }^{8,9}$, G. Roellinghoff ${ }^{52}$, M. Rongen ${ }^{39}$, C. Rott ${ }^{49}, 52$, T. Ruhe ${ }^{23}$, D. Ryckbosch ${ }^{29}$, D. Rysewyk $\mathrm{Cantu}^{24}$, I. Safa ${ }^{14,38}$, J. Saffer ${ }^{32}$, S. E. Sanchez Herrera ${ }^{24}$, A. Sandrock ${ }^{23}$, J. Sandroos ${ }^{39}$, M. Santander ${ }^{54}$, S. Sarkar ${ }^{44}$, S. Sarkar ${ }^{25}$, K. Satalecka $^{59}$, M. Scharf ${ }^{1}$, M. Schaufel ${ }^{1}$, H. Schieler ${ }^{31}$, S. Schindler ${ }^{26}$, P. Schlunder ${ }^{23}$, T. Schmidt ${ }^{19}$, A. Schneider ${ }^{38}$, J. Schneider ${ }^{26}$, F. G. Schröder ${ }^{31,42}$, L. Schumacher ${ }^{27}$, G. Schwefer ${ }^{1}$, S. Sclafani ${ }^{45}$, D. Seckel ${ }^{42}$, S. Seunarine ${ }^{47}$, A. Sharma ${ }^{57}$, S. Shefali ${ }^{32}$, M. Silva $^{38}$, B. Skrzypek ${ }^{14}$, B. Smithers ${ }^{4}$, R. Snihur ${ }^{38}$, J. Soedingrekso ${ }^{23}$, D. Soldin ${ }^{42}$, C. Spannfellner ${ }^{27}$, G. M. Spiczak ${ }^{47}$, C. Spiering ${ }^{59,}$, 61 , J. Stachurska $^{59}$, M. Stamatikos ${ }^{21}$, T. Stanev ${ }^{42}$, R. Stein ${ }^{59}$, J. Stettner ${ }^{1}$, A. Steuer ${ }^{39}$, T. Stezelberger ${ }^{9}$, T. Stürwald ${ }^{58}$, T. Stuttard ${ }^{22}$, G. W. Sullivan $^{19}$, I. Taboada ${ }^{6}$, F. Tenholt ${ }^{11}$, S. Ter-Antonyan ${ }^{7}$, S. Tilav ${ }^{42}$, F. Tischbein ${ }^{1}$, K. Tollefson $^{24}$, L. Tomankova ${ }^{11}$, C. Tönnis ${ }^{53}$, S. Toscano $^{12}$, D. Tosi $^{38}$, A. Trettin ${ }^{59}$, M. Tselengidou ${ }^{26}$, C. F. Tung ${ }^{6}$, A. Turcati ${ }^{27}$, R. Turcotte ${ }^{31}$, C. F. Turley ${ }^{56}$, J. P. Twagirayezu ${ }^{24}$, B. $\mathrm{Ty}^{38}$, M. A. Unland Elorrieta ${ }^{41}$, N. Valtonen-Mattila ${ }^{57}$, J. Vandenbroucke ${ }^{38}$, N. van Eijndhoven ${ }^{13}$, D. Vannerom ${ }^{15}$, J. van Santen $^{59}, \mathrm{~S}$.

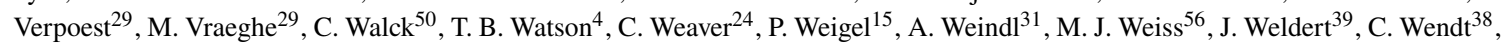
J. Werthebach ${ }^{23}$, M. Weyrauch ${ }^{32}$, N. Whitehorn ${ }^{24,35}$, C. H. Wiebusch ${ }^{1}$, D. R. Williams ${ }^{54}$, M. Wolf ${ }^{27}$, K. Woschnagg ${ }^{8}$, G. Wrede ${ }^{26}$, J.

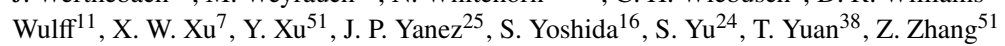

${ }^{1}$ III. Physikalisches Institut, RWTH Aachen University, D-52056 Aachen, Germany

2 Department of Physics, University of Adelaide, Adelaide, 5005, Australia

${ }^{3}$ Dept. of Physics and Astronomy, University of Alaska Anchorage, 3211 Providence Dr., Anchorage, AK 99508, USA

${ }^{4}$ Dept. of Physics, University of Texas at Arlington, 502 Yates St., Science Hall Rm 108, Box 19059, Arlington, TX 76019, USA

5 CTSPS, Clark-Atlanta University, Atlanta, GA 30314, USA

${ }^{6}$ School of Physics and Center for Relativistic Astrophysics, Georgia Institute of Technology, Atlanta, GA 30332, USA

${ }^{7}$ Dept. of Physics, Southern University, Baton Rouge, LA 70813, USA

${ }^{8}$ Dept. of Physics, University of California, Berkeley, CA 94720, USA

${ }^{9}$ Lawrence Berkeley National Laboratory, Berkeley, CA 94720, USA

${ }^{10}$ Institut für Physik, Humboldt-Universität zu Berlin, D-12489 Berlin, Germany

${ }^{11}$ Fakultät für Physik \& Astronomie, Ruhr-Universität Bochum, D-44780 Bochum, Germany

12 Université Libre de Bruxelles, Science Faculty CP230, B-1050 Brussels, Belgium

13 Vrije Universiteit Brussel (VUB), Dienst ELEM, B-1050 Brussels, Belgium

14 Department of Physics and Laboratory for Particle Physics and Cosmology, Harvard University, Cambridge, MA 02138, USA

15 Dept. of Physics, Massachusetts Institute of Technology, Cambridge, MA 02139, USA 
${ }^{16}$ Dept. of Physics and Institute for Global Prominent Research, Chiba University, Chiba 263-8522, Japan

${ }^{17}$ Department of Physics, Loyola University Chicago, Chicago, IL 60660, USA

${ }^{18}$ Dept. of Physics and Astronomy, University of Canterbury, Private Bag 4800, Christchurch, New Zealand

${ }^{19}$ Dept. of Physics, University of Maryland, College Park, MD 20742, USA

${ }^{20}$ Dept. of Astronomy, Ohio State University, Columbus, OH 43210, USA

${ }^{21}$ Dept. of Physics and Center for Cosmology and Astro-Particle Physics, Ohio State University, Columbus, OH 43210, USA

${ }^{22}$ Niels Bohr Institute, University of Copenhagen, DK-2100 Copenhagen, Denmark

${ }^{23}$ Dept. of Physics, TU Dortmund University, D-44221 Dortmund, Germany

${ }^{24}$ Dept. of Physics and Astronomy, Michigan State University, East Lansing, MI 48824, USA

${ }^{25}$ Dept. of Physics, University of Alberta, Edmonton, Alberta, Canada T6G 2E1

${ }^{26}$ Erlangen Centre for Astroparticle Physics, Friedrich-Alexander-Universität Erlangen-Nürnberg, D-91058 Erlangen, Germany

${ }^{27}$ Physik-department, Technische Universität München, D-85748 Garching, Germany

${ }^{28}$ Département de physique nucléaire et corpusculaire, Université de Genève, CH-1211 Genève, Switzerland

${ }^{29}$ Dept. of Physics and Astronomy, University of Gent, B-9000 Gent, Belgium

${ }^{30}$ Dept. of Physics and Astronomy, University of California, Irvine, CA 92697, USA

${ }^{31}$ Karlsruhe Institute of Technology, Institute for Astroparticle Physics, D-76021 Karlsruhe, Germany

${ }^{32}$ Karlsruhe Institute of Technology, Institute of Experimental Particle Physics, D-76021 Karlsruhe, Germany

${ }^{33}$ Dept. of Physics, Engineering Physics, and Astronomy, Queen's University, Kingston, ON K7L 3N6, Canada

${ }^{34}$ Dept. of Physics and Astronomy, University of Kansas, Lawrence, KS 66045, USA

${ }^{35}$ Department of Physics and Astronomy, UCLA, Los Angeles, CA 90095, USA

${ }^{36}$ Department of Physics, Mercer University, Macon, GA 31207-0001, USA

${ }^{37}$ Dept. of Astronomy, University of Wisconsin-Madison, Madison, WI 53706, USA

${ }^{38}$ Dept. of Physics and Wisconsin IceCube Particle Astrophysics Center, University of Wisconsin-Madison, Madison, WI 53706, USA

${ }^{39}$ Institute of Physics, University of Mainz, Staudinger Weg 7, D-55099 Mainz, Germany

${ }^{40}$ Department of Physics, Marquette University, Milwaukee, WI, 53201, USA

${ }^{41}$ Institut für Kernphysik, Westfälische Wilhelms-Universität Münster, D-48149 Münster, Germany

42 Bartol Research Institute and Dept. of Physics and Astronomy, University of Delaware, Newark, DE 19716, USA

${ }^{43}$ Dept. of Physics, Yale University, New Haven, CT 06520, USA

${ }^{44}$ Dept. of Physics, University of Oxford, Parks Road, Oxford OX1 3PU, UK

${ }^{45}$ Dept. of Physics, Drexel University, 3141 Chestnut Street, Philadelphia, PA 19104, USA

${ }^{46}$ Physics Department, South Dakota School of Mines and Technology, Rapid City, SD 57701, USA

${ }^{47}$ Dept. of Physics, University of Wisconsin, River Falls, WI 54022, USA

${ }^{48}$ Dept. of Physics and Astronomy, University of Rochester, Rochester, NY 14627, USA

${ }^{49}$ Department of Physics and Astronomy, University of Utah, Salt Lake City, UT 84112, USA

50 Oskar Klein Centre and Dept. of Physics, Stockholm University, SE-10691 Stockholm, Sweden

${ }^{51}$ Dept. of Physics and Astronomy, Stony Brook University, Stony Brook, NY 11794-3800, USA

52 Dept. of Physics, Sungkyunkwan University, Suwon 16419, Korea

${ }^{53}$ Institute of Basic Science, Sungkyunkwan University, Suwon 16419, Korea

${ }^{54}$ Dept. of Physics and Astronomy, University of Alabama, Tuscaloosa, AL 35487, USA

${ }^{55}$ Dept. of Astronomy and Astrophysics, Pennsylvania State University, University Park, PA 16802, USA

${ }^{56}$ Dept. of Physics, Pennsylvania State University, University Park, PA 16802, USA

${ }^{57}$ Dept. of Physics and Astronomy, Uppsala University, Box 516, S-75120 Uppsala, Sweden

${ }^{58}$ Dept. of Physics, University of Wuppertal, D-42119 Wuppertal, Germany

${ }^{59}$ DESY, D-15738 Zeuthen, Germany

${ }^{60}$ Università di Padova, I-35131 Padova, Italy

${ }^{61}$ National Research Nuclear University, Moscow Engineering Physics Institute (MEPhI), Moscow 115409, Russia

${ }^{62}$ Earthquake Research Institute, University of Tokyo, Bunkyo, Tokyo 113-0032, Japan

\section{Acknowledgements}

USA - U.S. National Science Foundation-Office of Polar Programs, U.S. National Science Foundation-Physics Division, U.S. National Science Foundation-EPSCoR, Wisconsin Alumni Research Foundation, Center for High Throughput Computing (CHTC) at the University of Wisconsin-Madison, Open Science Grid (OSG), Extreme Science and Engineering Discovery Environment (XSEDE), Frontera computing project at the Texas Advanced Computing Center, U.S. Department of Energy-National Energy Research Scientific Computing Center, Particle astrophysics research computing center at the University of Maryland, Institute for Cyber-Enabled Research at Michigan State University, and Astroparticle physics computational facility at Marquette University; Belgium - Funds for Scientific Research (FRS-FNRS and FWO), FWO Odysseus and Big Science programmes, and Belgian Federal Science Policy Office (Belspo); Germany - Bundesministerium für Bildung und Forschung (BMBF), Deutsche Forschungsgemeinschaft (DFG), Helmholtz Alliance for Astroparticle Physics (HAP), Initiative and Networking Fund of the Helmholtz Association, Deutsches Elektronen Synchrotron (DESY), and High Performance Computing cluster of the RWTH Aachen; Sweden - Swedish Research Council, Swedish Polar Research Secretariat, Swedish National Infrastructure for Computing (SNIC), and Knut and Alice Wallenberg Foundation; Australia - Australian 
Research Council; Canada - Natural Sciences and Engineering Research Council of Canada, Calcul Québec, Compute Ontario, Canada Foundation for Innovation, WestGrid, and Compute Canada; Denmark - Villum Fonden and Carlsberg Foundation; New Zealand Marsden Fund; Japan - Japan Society for Promotion of Science (JSPS) and Institute for Global Prominent Research (IGPR) of Chiba University; Korea - National Research Foundation of Korea (NRF); Switzerland - Swiss National Science Foundation (SNSF); United Kingdom - Department of Physics, University of Oxford. 